\title{
In situ polymerisation as a route towards transparent nanocomposites: Time-resolved light and neutron scattering experiments
}

\author{
Mirko Saric ${ }^{\mathrm{a}}$, Hervé Dietsch ${ }^{\mathrm{b}}$, Peter Schurtenberger ${ }^{\mathrm{a}, *}$ \\ ${ }^{a}$ Department of Physics, Soft Condensed Matter Group, University of Fribourg, Chemin du musée 3, CH-1700 Fribourg, Switzerland \\ ${ }^{\mathrm{b}}$ Laboratory for Nanoscale Materials Science EMPA - Materials Science \& Technology, EMPA, CH-8600 Dübendorf, Switzerland
}

\begin{abstract}
We describe the formation of a highly transparent nanocomposite using an in situ polymerisation of monodisperse, $50 \mathrm{~nm}$ diameter, colloidal silica coated with 3-(trimethoxysilyl)propyl methacrylate (TPM) dispersed in methyl methacrylate (MMA). We demonstrate that it is possible to follow the polymerisation process in a completely non-invasive way using time-resolved dynamic light scattering and small-angle neutron scattering. These experiments allow us to obtain quantitative information on the time dependence of the resulting mechanical and structural properties during the synthesis. We observe a qualitative change between diffusive and subdiffusive motion for the dispersed nanoparticles at the gel point, in agreement with the behaviour of other colloidal systems undergoing a sol-gel transition. The small-angle neutron scattering experiments clearly demonstrate that the initial liquid-like order of the nanoparticles is maintained during the polymerisation process, and that there is no indication for particle aggregation. This information is confirmed by TEM experiments of the final nanocomposite.
\end{abstract}

Keywords: Nanocomposite; In situ polymerisation; Dynamic light scattering; Small-angle neutron scattering

\section{Introduction}

Nanostructured organic-inorganic hybrid systems represent an exiting class of materials. The combination of nanoscale inorganic moieties with organic polymers allows us to combine the properties of nanoparticles and polymers and thus to design materials with enhanced or even completely new properties. Another important feature is the possibility of tuning the structure and morphology on the nanometric scale, which opens the door for innovative applications in both advanced and conventional technology fields. Selected examples for potential applications are the design of new components in electronic and optoelectronic devices, bioencapsulation or as active substrates in chromatography and catalysis. The unique properties of these materials, such as their improved mechanical [1], thermal [2-5], electrical [6], magnetic [7], photochemical [8], and catalytic [9] performance in comparison to pure organic polymers as well as their high optical transparency [10] has created enormous

\footnotetext{
* Corresponding author. Tel.: +4126300 9115; fax: +41 263009747 . E-mail address: peter.schurtenberger@unifr.ch (P. Schurtenberger).
}

interest among materials scientists and engineers. However, the control of the mixing between the two dissimilar phases remains a challenging task, and there still is a lack of data on structureproperty relationships at a nanoscale level [11]. We have thus started a systematic study in which we combine synthetic activities with an application of time-resolved scattering methods in order to understand and improve the formation mechanism of polymer-colloid nanocomposites.

A major obstacle on the way towards versatile nanocomposites with tailor-made properties arises from the poor miscibility of the two dissimilar phases. The properties of particulatefilled composites are generally determined by the component properties, composition, structure (spatial distribution), particle-particle interaction and particle-matrix interaction. Although it is well accepted that full dispersion (disaggregation) of the particles is of crucial importance for the performance of the composites, there is still no quantitative method to predict and/or measure the dispersability of the filler particles in the polymer matrix. The extent to which particles agglomerate depends both on the processing conditions (shear forces) as well as on the balance between the attractive and repulsive forces among the particles and the matrix. Depending on the chemical interac- 


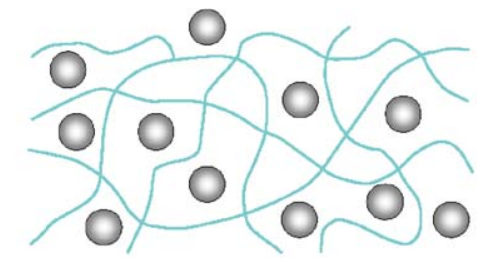

(a)

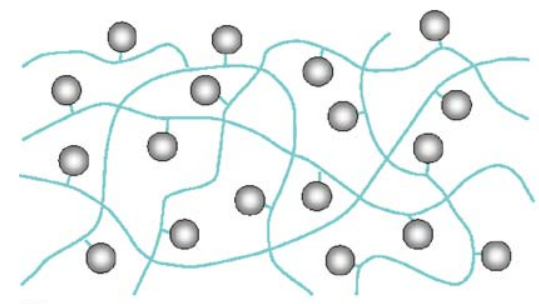

(b)

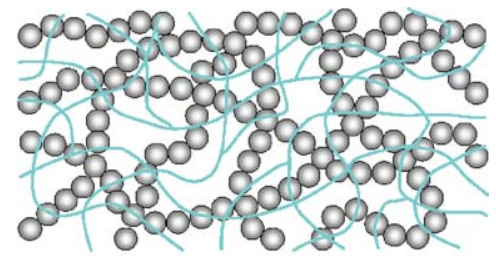

(b)

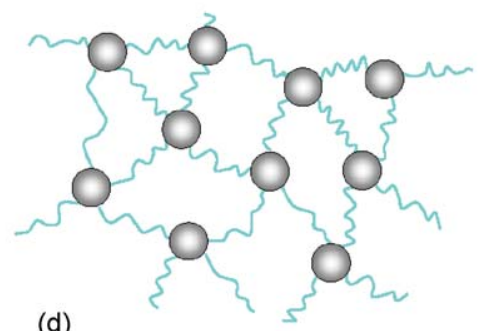

(d)

Fig. 1. Selected examples of stable polymer-colloid nanocomposites, where the particles (a) are present in a stable dispersion, (b) form an interpenetrating network, (c) are chemically linked to the polymer matrix, or (d) act as crosslinkers.

tion between the species, one can differ between materials with strong (covalent, coordination or ionic) or weak (van der waals, hydrogen-bonds) interactions [12,13].

In principle, there are various ways of creating stable and fully dispersed polymer-colloid nanocomposites with different properties, and Fig. 1 shows some schematic drawings of selected examples. In practice, a simple dispersion of inorganic nanoparticles in a polymer matrix as depicted in Fig. 1a is seldom feasible since polymers and inorganic fillers often possess incompatible segments leading to micro- and/or macrophase separation and a concomitant clustering on various length scales [14]. There are several possibilities in order to avoid these phenomena: (1) to attach an inert organic layer covalently to the surface of the particle in order to enhance the compatibility of the polymer-particle interface [16]; (2) to encapsulate the nanoparticles by emulsion polymerisation [10]; (3) to attach functional or initiating groups which allow a covalent linkage to the polymer matrix, or (4) to graft polymer chains from the particle surface which leads to a steric stabilization of the nanoparticles and enhanced stability of the resulting material $[17,18]$.

Here we now describe the use of method 3, where we functionalize nanoparticles and thus make them available as crosslinkers for the creation of a polymer gel through an in situ polymerisation. This should then lead to a situation as schematically shown in Fig. 1d. We use a poly(methyl methacrylate)
(PMMA) based system. PMMA belongs to the important species of polyacrylic and methacrylic esters, and possesses high optical transparency, exceptional mechanical strength and form stability as some of its most important materials properties. In combination with nanoparticles, it is in addition possible to decrease the gas permeability, and to enhance the physical performance and heat resistance without diminishing the optical transparency [10]. Optically clear composites of silica-PMMA have been prepared by using silica particles that are much smaller than the wavelength of visible light or by matching the refractive index of the components $[19,20]$. However, the transparency of the composite decreases dramatically if aggregation occurs, since clusters of nanoparticles strongly scatter visible light. Several routes towards stable PMMA-silica-based nanocomposites have been followed in the past. In order to improve the compatibility of silica and vinyl polymer matrices, silane coupling agents have been grafted on colloidal silica surfaces [21,22]. PMMAlayered silicate nanocomposites were also prepared by in situ suspension and emulsion polymerisation [10]. Organic polymer hybrids can also be synthesized using in-situ sol-gel methods where TEOS is hydrolyzed and condensed in the presence of the chosen polymer [15].

Our approach for the nanocomposite preparation was to use surface modified silica nanoparticles suspended in a monomer solvent, followed by an in situ polymerisation (Fig. 2) [23].
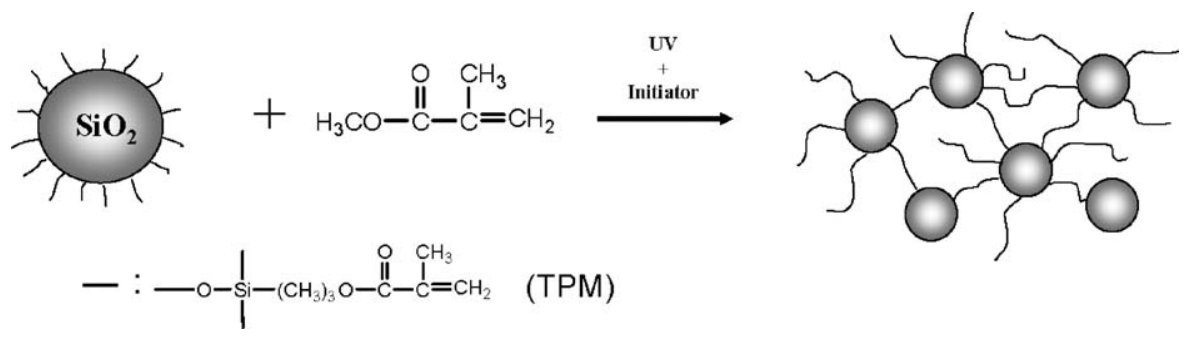

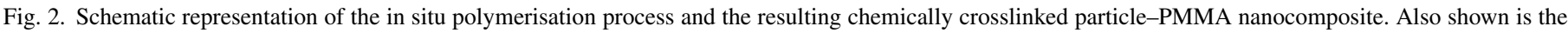
surface modification of the silica particles with TPM. 
We used 3-(trimethoxysilyl)-propylmethacrylate (TPM) surface modified silica nanoparticles that have a radius of $25 \mathrm{~nm}$. For the radical polymerisation, we did not use an additional organic solvent, the monomer undertakes this function as well. After the reaction no further purification from the solvent is necessary, and a possible agglomeration due to the drying processes is avoided. The polymerisation process is initialized by an initiator and UV-irradiation. The particles then act as crosslinkers, and any potential clustering and micro- or macrophase separation due to depletion effects arising from the growing polymer chains can be avoided. The resulting nanocomposites thus remain highly transparent.

In this paper, we describe the use of dynamic light scattering (DLS) in order to monitor the Brownian motion of the TPM-silica particles during the polymerisation process. DLS is a powerful tool to scan thermal fluctuations of dispersed probe particles that are related to the (local) viscoelastic properties of the system [24]. In addition, we describe time-resolved SANS experiments that enable us to determine the evolution of the static structure and the possible formation of particle clusters during the in situ polymerisation. The combination of these two methods allows us to gain detailed information about the time evolution of the structure and dynamics of the medium on a microscopic level. It thus becomes possible to follow the polymerisation process in a completely non-invasive and timeresolved way, and we obtain access to the kinetics and dynamics of the production process of nanocomposite materials and the resulting mechanical properties.

\section{Experimental}

\subsection{Nanocomposite preparation}

3-(Trimethoxysilyl)-propylmethacrylate grafted silica nanoparticles with an averaged diameter of $50 \mathrm{~nm}$ were prepared in methanol as described before [23] and transferred to methyl methacrylate by dialysis. The dispersions were polymerised in round $10 \mathrm{~mm}$ tubes. The system containing the colloidal silica dispersion and $0.2 \mathrm{wt} . \%$ of the initiator (Irgacure 184, Ciba SC) was polymerised at ambient temperature over $2 \mathrm{~h}$ using an UV-lamp (UV SPOT 400T, Dr. Hönle, Germany) with a H2 UV-filter in order to reduce heating via Infrared absorption. The tube was rotated around its horizontal axis to guarantee complete mixing and equal UV-irradiation. The DLS experiments were preformed in situ with the same sample holder. The samples for SANS experiments were prepared in standard Hellma quartz cells with a path length of $2 \mathrm{~mm}$ instead of round $10 \mathrm{~mm}$ tubes using the same polymerisation protocol.

\subsection{Nanocomposite characterization}

The time-resolved DLS experiments were carried out with an ALV DLS/SLS-5000 compact goniometer system with an ALV5000 fast correlator and an argon-ion laser source (Coherent Innova 300) operating at a wavelength of $514.5 \mathrm{~nm}$. For the timeresolved in situ measurements with polymerising samples we worked at a single scattering angle of $\theta=60^{\circ}$ and at a temperature of $T=20^{\circ} \mathrm{C}$

The measured intensity correlation function is defined as $g_{2}(\tau)=\langle I(t) I(t+\tau)\rangle /\langle I(t)\rangle^{2}$. The experimental correlation function is related to the normalized field autocorrelation function $g_{1}(t)$ :

$g_{2}(\tau)=1+A\left[g_{1}(\tau)\right]^{2}$

where $A$ is a constant that depends on the experimental setup. The mean-square displacement (MSD) of the particles can be calculated from $g_{1}(\tau)[25]$ :

$g_{1}(\tau)=\exp \left[-\frac{q^{2}}{6}\left\langle\Delta r^{2}(\tau)\right\rangle\right]$.

The SANS measurements were performed at the SANS I instrument of the Swiss Neutron Source SINQ at the Paul Scherer Institute and covered a range of scattering vectors $q=0.01-1.5 \mathrm{~nm}^{-1}$. Three settings were used, with combinations of sample-detector distance and neutron wavelength of $4.5 \mathrm{~m}$ and $0.8 \mathrm{~nm}, 12 \mathrm{~m}$ and $8 \mathrm{~nm}$, and $20.27 \mathrm{~m}$ and $1.267 \mathrm{~nm}$, respectively. The samples were measured in standard Hellma quartz cells with a path length of $2 \mathrm{~mm}$. The SANS data was converted to an absolute scale with a $1 \mathrm{~mm}$ thick water sample as a secondary standard.

The polymerised nanocomposites were also investigated using transmission electron microscopy (TEM). A sample of a $\mathrm{SiO}_{2}$-PMMA composite film containing $13.3 \mathrm{wt} . \%$ (6.2 vol. \%) of $50.8 \mathrm{~nm}$ diameter particles was cut with a diamond blade "Cryotim 45" from Diatome, Switzerland (www.diatome.ch). The thickness was then reduced to a nominal value of $50 \mathrm{~nm}$ using an ultramicrotome Leica UCT equipped with an "ultra $35^{\circ}$ " knife at a cutting speed of $1 \mathrm{~mm} / \mathrm{s}$. The thus obtained thin section was then transferred onto a 200 mesh carbon grid and investigated using brightfield TEM images made on a CM100 Philips microscope operated at $80 \mathrm{kV}$.

\section{Results and discussion}

Time-resolved DLS experiments were performed on a dispersion of $3.3 \mathrm{wt}$ \% TPM-silica nanoparticles in a MMA monomer solvent during radical polymerisation at a fixed scattering angle of $60^{\circ}$ and a temperature of $20^{\circ} \mathrm{C}$. The corresponding intensity autocorrelation functions are presented in Fig. 3. Due to the very high scattering contrast of the $\mathrm{SiO}_{2}$ particles, the dominant contribution to the scattered light intensity arises from the nanoparticles, and the resulting correlation functions reflect the particle dynamics even after polymerisation. For the initial suspension, i.e. before the polymerisation is initiated at $t=0$, the TPM-silica nanoparticles are able to undergo free Brownian motion characterized by a single exponential decay for $g_{2}(\tau) \sim \exp \left(-\tau / \tau_{\mathrm{c}}\right)$. The characteristic decay time $\tau_{\mathrm{c}}$ is then given by the product of the diffusion coefficient $D_{0}$ and the magnitude of the square of the scattering vector, i.e., by $\tau_{\mathrm{c}}=\left(D_{0} q^{2}\right)^{-1}$. The addition of the initiator and the irradiation with UV-light then triggers the radical polymerisation of the monomer. Poly(methyl methacrylate)-polymer chains now start to grow freely in the 


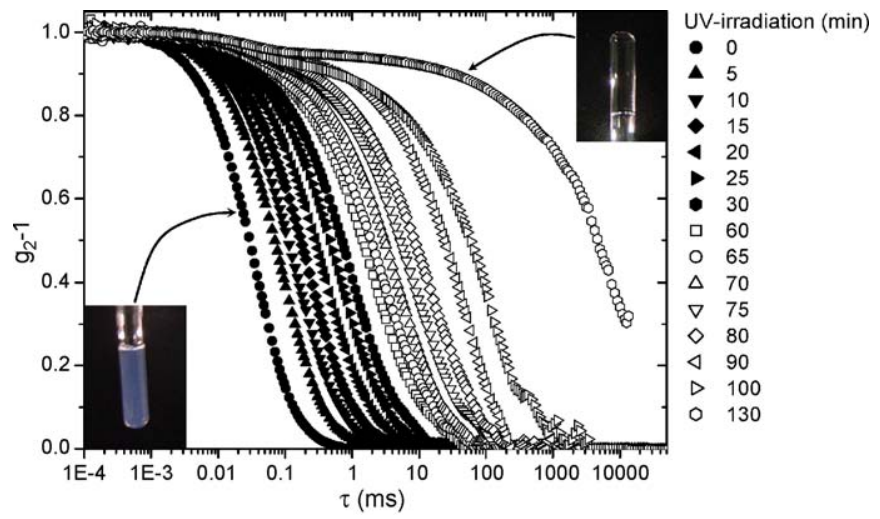

Fig. 3. Normalized intensity correlation function of $3.3 \mathrm{wt} . \%$ TPM-silica nanoparticles measured at a scattering angle $\theta=60^{\circ}$ at different polymerisation times. Solid symbols indicate Brownian motion of nanoparticles, whereas open symbols indicate subdiffusive motion. Also shown as insets are photographs of the initial and final samples.

monomer solution, but also covalently bonded to the particles surface. This is schematically illustrated in Fig. 4a. With ongoing UV-irradiation the correlation functions thus shift towards longer decay times, indicating that the particle motion slows down gradually. This is due to the fact that the polymers attached to the particles increase the hydrodynamic radius of the colloids, and the growing polymer chain length in the matrix results in an increase of the background viscosity.

However, as the polymerisation time increases even further, we observe a qualitative change in the shape of the correlation functions. The characteristic decay is no longer single exponential, but appears to become stretched, and a plateau with a subsequent second decay develops. The height of this plateau increases with time due to the network formation and crosslinking of the particles. This dramatic change in the particle dynamics is accompanied by a tremendous increase of the viscosity of the sample and a subsequent transition to a solid-like state. However, the sample remains fully transparent at all times as demonstrated by the two photographs of the initial and final sample shown as insets in Fig. 3, where the latter also demonstrates the solid-like character of the final nanocomposite.

For a quantitative understanding of the temporal evolution of the microscopic particle dynamics in the course of the polymerisation process, we analyzed the particle mean square displacement $\left\langle\Delta r^{2}(\tau)\right\rangle$ as calculated from all correlation functions $g_{2}-1$ shown in Fig. 3 using Eq. (2). The data shown in Fig. $4 b$ and c demonstrate that the nanoparticles initially, i.e. without UVirradiation, show a behaviour typical for Brownian diffusion over the whole accessible time range. This is characterized by a linear $\left\langle\Delta r^{2}(\tau)\right\rangle$, where $\left\langle\Delta r^{2}(\tau)\right\rangle$ is given by $\left\langle\Delta r^{2}(\tau)\right\rangle=6 D_{0} \tau$. With increasing duration of the UV-irradiation the polymer chains grow and start to form a network. The initial short time behaviour changes qualitatively from diffusive to subdiffusive motion and can be described by a power law $\left\langle\Delta r^{2}(\tau)\right\rangle \propto \tau^{p}$, where the exponent $p$ decreases from $p \approx 1$ to a value of $p \approx 0.7$ during the network formation. At the same time, the particle motion becomes constrained, and a plateau in the MSD develops.
The MSD obtained from samples at longer polymerisation times, where the formation of a viscoelastic solid is induced, all show the same qualitative behaviour. Their initial time dependence is well described by a stretched exponential of the form

$\left\langle\Delta r^{2}(\tau)\right\rangle=\delta^{2}\left[1-\mathrm{e}^{-\left(\tau / \tau_{\mathrm{c}}\right)^{p}}\right]$,

indicating that for the silica colloids the particle excursion is restricted to a maximum MSD $\delta^{2}$. Similar expressions for the short time dynamics of colloidal particles have for example been found for colloidal gels [24,26,27] or tracer particles in crosslinked polymer gels [28] or actin networks [29]. The good agreement between the experimental data and the description of constrained subdiffusive motion of the nanoparticles given by Eq. (3) is demonstrated in Fig. 5, where we plot a master curve obtained by normalizing $\tau$ with $\tau_{\mathrm{c}}$ and $\left\langle\Delta r^{2}(\tau)\right\rangle$ by $\delta^{2}$.

It has already been previously pointed out that the constrained motion of tracer particles in viscoelastic solids or liquids must reflect the elasticity of the background medium, and this has been used successfully in the application of the so-called microrheology concept to the characterization of complex fluids and solids [24]. Therefore, we can use the MSD and in particular the crossover from Brownian to constrained subdiffusive motion in order to obtain additional information about the network formation in the nanocomposite. This is illustrated in Fig. 6, where we plot the temporal evolution of the exponent $p$ and the characteristic relaxation time $\tau_{\mathrm{c}}$ from an analysis of the MSD shown in Fig. 4b.

During the first $30 \mathrm{~min}, p$ decreases only slightly from a value of 1 indicating Brownian motion, but $\tau_{\mathrm{c}}$ increases dramatically by almost an order of magnitude as a result of the slowing down of the particle motion in the growing polymer solution. After this initial polymerisation period, the situation changes completely. The relaxation time now starts to decrease strongly, while $p$ continues to decrease to a final value of 0.7 . This reflects the fact that the nature of the nanoparticle dynamics now qualitatively changes. The particles no longer undergo Brownian motion in a viscous polymer fluid, but become chemically crosslinked to a polymer network. Therefore, $\tau_{\mathrm{c}}$ no longer reflects the diffusion coefficient of the polymer-coated particles, but rather the local viscoelastic properties of the polymer network. As pointed out previously, both $\tau_{\mathrm{c}}$ and $\delta^{2}$ are linked to the elastic modulus of the resulting gel. A simple argument would be that the work necessary for a particle to move over a distance $\delta$ is equal to the thermal energy $k T$ of the particle. This allows us to at least estimate the elastic shear modulus $G$ via

$G=\frac{k T}{6 \pi a \delta^{2}}$

where $G$ has the meaning of a local shear modulus and a is the particle radius [29-31]. If we use the data summarized in Fig. 4b for the longest polymerisation time of $130 \mathrm{~min}$, we obtain a value for $\delta^{2} \approx 480 \mathrm{~nm}^{2}$, which yields a shear modulus of $G \approx 18 \mathrm{~Pa}$. This is quite reasonable in view of the fact that at this point the system is far from being fully polymerised yet. The fact that we have not yet reached the end of the polymerisation 

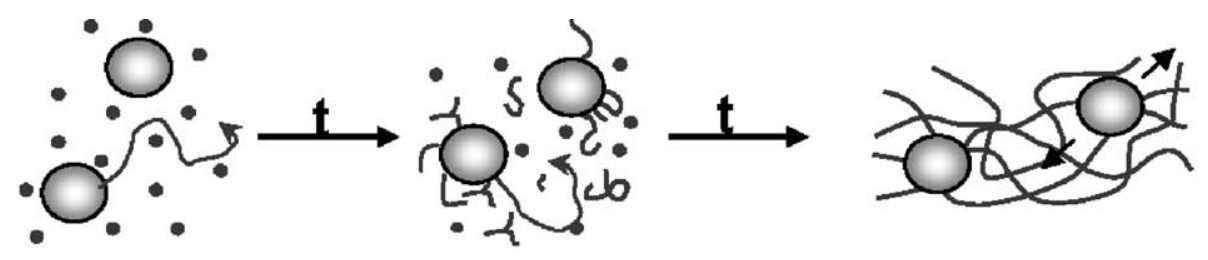

free Brownian motion

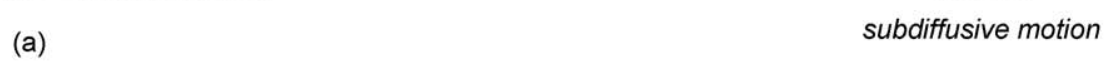

(a)
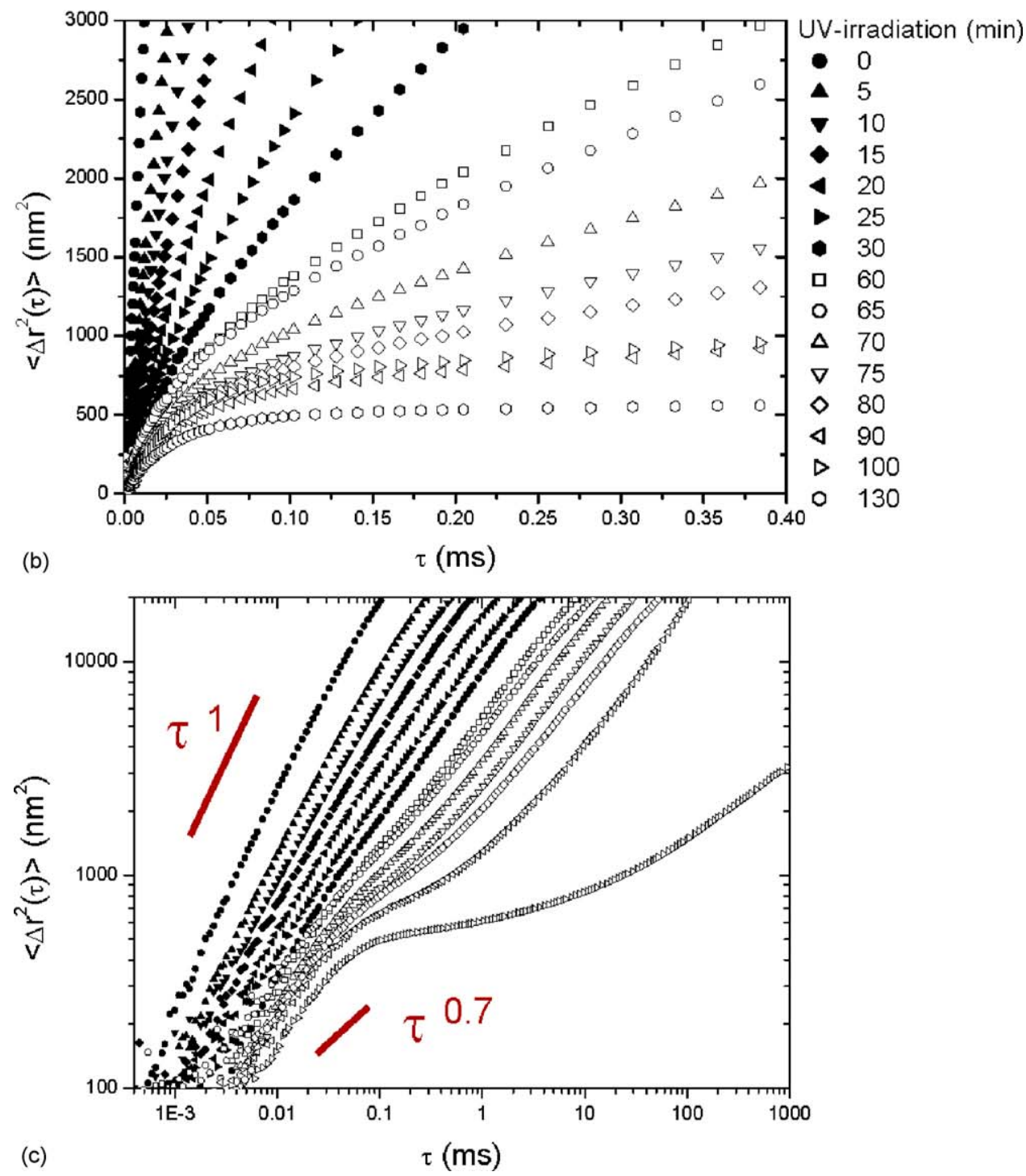

Fig. 4. (a) Schematic representation of the UV-initiated polymerisation process, where the particles are initially freely suspended in the monomer solvent. Polymerisation then results in growing polymers both in the melt as well as covalently attached to the particle surface. The functionalized particles act as crosslinkers, and a fully crosslinked polymer gel is formed. (b) Initial time dependence of the mean-square displacement of 3.3 wt.\% nanoparticles suspended in MMA monomer solvent at different polymerisation times at $20^{\circ} \mathrm{C}$. (c) Complete representation of the measured MSD over the full range of accessible time scales for all data shown in (b).

process is also visible from the shape of the MSD when plotting the full range of accessible time scales (Fig. 4c). Here, we see that the MSD shows an apparent plateau, but that a second very slow relaxation process is still present, indicating that the system is rather viscoelastic and not completely solid or glasslike.
The DLS experiments have allowed us to achieve a noninvasive characterization of the particle dynamics during the formation of a silica-PMMA nanocomposite via in situ polymerisation. However, they do not provide information about the degree of agglomeration that often occurs in nanocomposites. Therefore, we have conducted time-resolved SANS- 


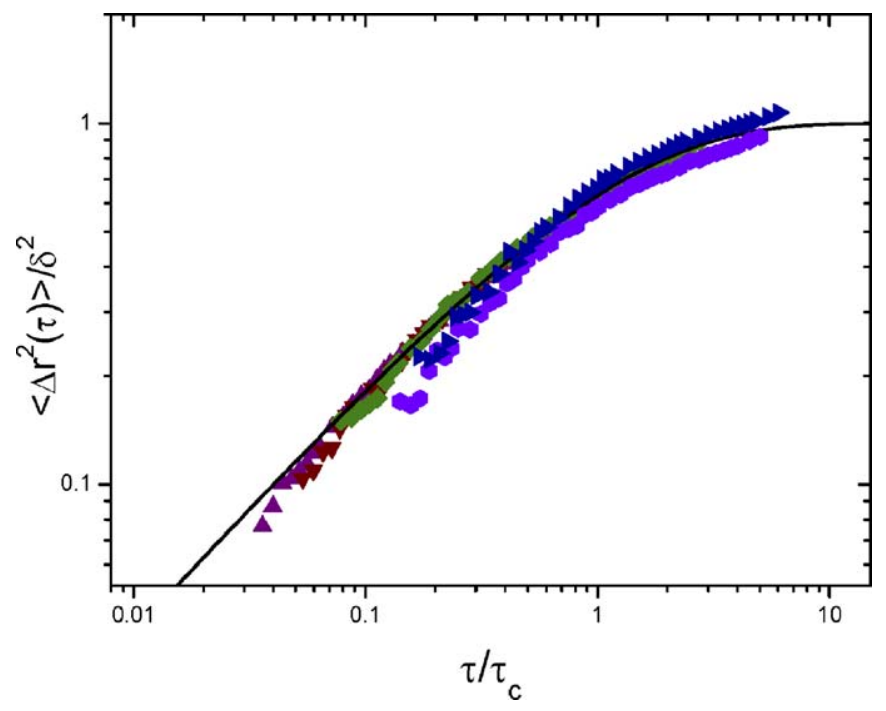

Fig. 5. Master curve $\left\langle\Delta r^{2}(t)\right\rangle / \delta^{2}$ vs. $t / \tau_{\chi}$ for the MSD obtained for samples at longer polymerisation times, i.e., where viscoelastic solids have formed.

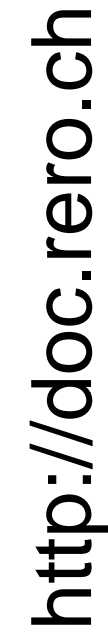

measurement during the in situ polymerisation of a nanocomposite with a high particle loading of $13.3 \mathrm{wt} . \%$ TPM-silica nanoparticles (Fig. 7a). The initial colloidal suspensions show a $q$-dependence typical for hard sphere colloids at moderate volume fractions. This becomes apparent from a determination of
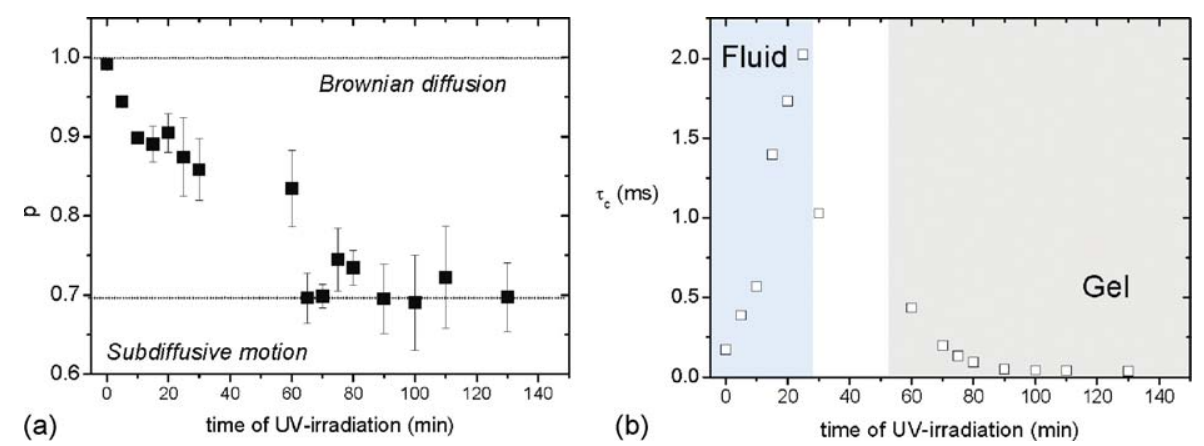

Fig. 6. Time evolution of parameter $p$ (a) and $\tau_{\mathrm{c}}$ (b) from an analysis of the MSD obtained for the $3.3 \mathrm{wt} . \%$ TPM-silica nanoparticles in MMA during radical polymerisation using Eq. (3).
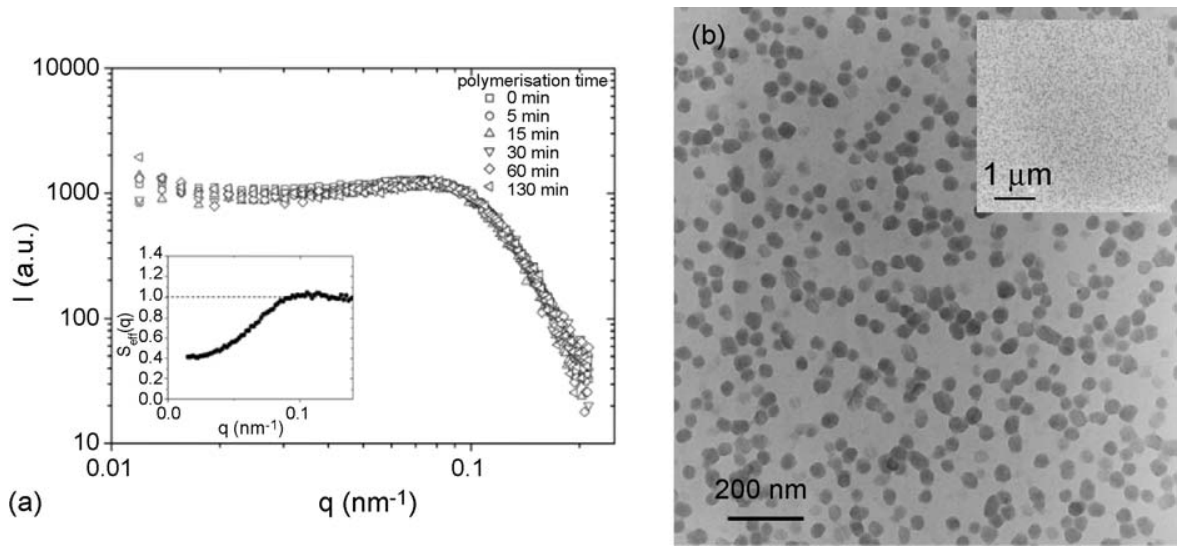

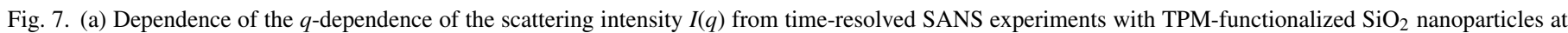
a concentration of $13 \mathrm{wt}$ \% dispersed in MMA at different polymerisation times. Also shown as an inset is the effective structure factor $S_{\text {eff }}(q)$ of the initial particle dispersion. (b) TEM micrographs of the final nanocomposite at two different magnifications. 
functionalization of the silica nanoparticles combined with an in situ polymerisation results in the formation of a stable nanocomposite with well-dispersed colloids is further supported from subsequent TEM measurements shown in Fig. 7b. This figure presents pictures with two different magnifications that clearly demonstrate the absence of inhomogeneities at all length scales.

\section{Conclusions}

The combination of functionalizing monodisperse silica nanoparticles with TPM and an in situ polymerisation of the monomer solvent results in the formation of completely stable and highly transparent nanocomposite materials up to particle concentrations of $13 \mathrm{wt} \%$. Time-resolved dynamic light scattering and small-angle neutron scattering experiments have allowed us to follow the in situ polymerisation of the nanocomposites in a completely non-invasive way. They provide a quantitative description of the particle dynamics during the polymerisation process and the subsequent formation of a chemically crosslinked polymer gel, and they have allowed us to assess the lack of agglomeration and absence of inhomogeneities. Moreover, DLS has yielded additional information on the development of the mechanical properties of the system as the polymers and network form. In principle, the microrheology approach will allow us to go beyond a simple determination of the modulus and arrive at a full time-resolved description of the frequency dependent storage and loss moduli [24]. While this is beyond the scope of this first study, it is worth pointing out the potential of these combined experiments in the investigation of the kinetics of polymerisation and structure formation due to the completely non-invasive character of the scattering experiments and the fact that we achieve structural characterization and temporal resolution on the required length and time scales.

\section{Acknowledgements}

The authors are grateful to Beat Keller for encouraging and stimulating discussions. We thank Nicolas Sary, UniFr, who performed the TEM experiments, and Helmut Gnägi, Diatome.ch, who kindly prepared the thin sections for the TEM samples. Financial support was provided by the Commission Suisse pour la Technologie et l'Innovation (CTI), Project no 6056.2. This work is based in part on experiments performed at the Swiss spallation neutron source SINQ, Paul Scherrer Institute, Villigen, Switzerland. We are grateful for the neutron beam time and we acknowledge the help of our local contacts J. Kohlbrecher and S. van Petegem.

\section{References}

[1] A. Okada, A. Usuki, Mater. Sci. Eng. 3 (1995) 109.

[2] J.W. Gilman, Appl. Clay Sci. 15 (1999) 31.

[3] J.W. Gilman, C.L. Jackson, A.B. Morgan, R. Harris Jr., E. Manias, E.P. Giannelis, M. Wuthenow, D. Hilton, S.H. Phillips, Chem. Mater. 12 (2000) 1866.

[4] D. Porter, E. Metcalfe, M.J.K. Thomas, Fire Mater. 24 (2000) 45.

[5] M. Zanetti, S. Lomakin, G. Camino, Macromol. Mater. Eng. 279 (2000) 1.

[6] S.P. Armes, Polym. News 20 (1995) 233.

[7] D.Y. Godovski, Adv. Polym. Sci. 119 (1995) 79.

[8] G. Wirnsberger, B.J. Scott, B.F. Chmelka, G.D. Stucky, Adv. Mater. 12 (2000) 1450.

[9] A. Hanprasopwattana, S. Srinivasan, A.G. Sault, A.K. Datye, Langmuir 12 (1996) 3173.

[10] X. Huang, W.J. Brittain, Macromolecules 34 (2001) 3255.

[11] P.B. Messersmith, S.I. Stupp, J. Mater. Res. 7 (1992) 2559

[12] P. Judeinstein, C. Sanchez, J. Mater. Chem. 6 (1996) 511

[13] U. Schubert, N. Hüsing, Synthesis of Inorganic Materials, Wiley-VCH, Weinheim, 2000.

[14] F.S. Bates, G.H. Fredrickson, Annu. Rev. Phys. Chem. 41 (1990) 559.

[15] Y. Chujo, T. Saegusa, Adv. Polym. Sci. 100 (1992) 11.

[16] W.D. Hergeth, M. Peller, P. Hauptmann, Acta Polym. 37 (1986) 468.

[17] G. Kickelbick, U. Schubert, Monatsh. Chem. 132 (2001) 13.

[18] U. Schubert, Chem. Mater. 13 (2001) 3487.

[19] E.J.A. Pope, M. Asami, J.D. Mackenzie, J. Mater. Res. 4 (1989) 1018

[20] H. Lin, D.E. Day, J.O. Stoffer, Polym. Eng. Sci. 32 (1992) 344.

[21] N. Nishiyama, K. Horie, R. Schick, H. Ishida, Polym. Commun. 31 (1990) 380.

[22] A. Morikawa, H. Yamaguchi, M. Kakimoto, Y. Imai, Chem. Mater. 6(1994) 913.

[23] H. Dietsch, D. Lootens, B.A. Keller, Synthesis and rheological study of tailored monodisperse and monodistributed $\mathrm{SiO}_{2}$-PMMA composites, in preparation 2006.

[24] F. Scheffold, P. Schurtenberger, Soft Mater. 1 (2003) 139.

[25] B. Berne, R. Pecora, Dynamic Light Scattering: With Applications to Chemistry, Biology and Physics, Wiley, New York, 1976.

[26] A. Krall, D.A. Weitz, Phys. Rev. Lett. 80 (1998) 778.

[27] S. Romer, F. Scheffold, P. Schurtenberger, Phys. Rev. Lett. 85 (2000) 4980.

[28] P. Schurtenberger, H. Bissig, L. Rojas, R. Vavrin, A. Stradner, S. Romer, F. Scheffold, V. Trappe, Mesoscale phenomena in fluid systems, in: F. Case, P. Alexandridis (Eds.), AC01, 2003, pp 143-160.

[29] T. Narita, A. Knaebel, J.-P. Munch, M. Zrinyi, S.J. Candau, Macromol. Symp. 207 (2004) 17.

[30] F. Amblard, A.C. Maggs, B. Yurke, A.N. Pargellis, S. Leibler, Phys. Rev. Lett. 77 (1996) 4470.

[31] B. Schnurr, F. Gittes, F.C. MacKintosh, C.F. Schmidt, Macromolecules 30 (1997) 7781.

[32] N.F. Carnahan, K.E. Starling, J. Chem. Phys. 51 (1969) 635.

[33] D. Gazzillo, A. Giacometti, J. Chem. Phys. 113 (2000) 9837. 